\title{
Finite element modelling of cochlear electrical coupling
}

\author{
Paul D. Teal ${ }^{\text {a) }}$ \\ School of Engineering and Computer Science, Victoria University of Wellington, Kelburn Parade, \\ Wellington 6140, New Zealand \\ Guangjian Ni \\ Institute of Sound and Vibration Research, University of Southampton, Southampton, SO17 1BJ, \\ United Kingdom
}

(Received 14 March 2016; revised 23 August 2016; accepted 30 September 2016; published online 18 October 2016)

\begin{abstract}
The operation of each hair cell within the cochlea generates a change in electrical potential at the frequency of the vibrating basilar membrane beneath the hair cell. This electrical potential influences the operation of the cochlea at nearby locations and can also be detected as the cochlear microphonic signal. The effect of such potentials has been proposed as a mechanism for the non-local operation of the cochlear amplifier, and the interaction of such potentials has been thought to be the cause of the broadness of cochlea microphonic tuning curves. The spatial extent of influence of these potentials is an important parameter for determining the significance of their effects. Calculations of this extent have typically been based on calculating the longitudinal resistance of each of the scalae from the scala cross sectional area, and the conductivity of the lymph. In this paper, the range of influence of the electrical potential is examined using an electrical finite element model. It is found that the range of influence of the hair cell potential is much shorter than the conventional calculation, but is consistent with recent measurements.
\end{abstract}

(C) 2016 Acoustical Society of America. [http://dx.doi.org/10.1121/1.4964897]

[BLM]

Pages: 2769-2779

\section{INTRODUCTION}

The hair cells of the cochlea provide an essential function in the transduction of sound; the inner hair cells activate neurons, while the outer hair cells (OHCs) provide mechanical amplification of the sounds. For both inner hair cells and OHCs, their function arises from a flow of ions modulated by mechanical deflection of their stereocilia. This ion flow causes a change in the electrical potential near the cells.

This change in electrical potential propagates as an electrical field and can influence the operation of the cochlea outside of the hair cell that caused the change. Electrodes both inside and outside of the cochlea can detect these changes, recording a signal known as the cochlear microphonic (Wever and Bray, 1930).

The effect of this propagation has been proposed as a mechanism for non-local operation of the cochlear amplifier (Dallos and Evans, 1995; Dimitriadas and Chadwick, 1999).

The frequency of the electric field is the frequency of the basilar membrane vibration at any given hair cell location. Destructive interference between potentials generated at different positions within the cochlea has been thought to be the cause of the broadness of cochlea microphonic tuning curves compared with mechanical basilar membrane tuning curves (Patuzzi, 1987).

The physical distance over which these potentials propagate is an important parameter for determining the significance of their effects. Calculations of this range has

${ }^{a) E l e c t r o n i c ~ m a i l: ~ p a u l . t e a l @ v u w . a c . n z ~}$ typically been based on calculating the longitudinal resistance of each of the scalae from the scala cross-sectional area, and the conductivity of the lymph. These calculations appear in Strelioff (1973), and many models of electrical interactions within the cochlea have used this as a basis (Ayat et al., 2014; Ayat and Teal, 2013; Honrubia et al., 1973; Mistrík et al., 2009; Ramamoorthy et al., 2007; Teal et al., 2011). Models based on this approach typically include "ladder" networks of resistors. These have been described as resistive cable models.

It has been suggested that the voltage decays exponentially with the distance from the active hair cell, with a decay constant or space constant (distance for voltage to decay by $1 / e$ of the original amplitude) of $0.5-4 \mathrm{~mm}$ (Geisler et al., 1990). Johnston et al. (1966) used measurements with electrodes to estimate this distance in the scala media as $2 \mathrm{~mm}$.

Another approach for modelling the propagation of electric fields was used by Chertoff et al. (2012) to examine propagation of the electric field to a far field electrode. This modelled each hair cell as multiple points of charge.

In this paper, the propagation of the electrical field in the cochlea is examined using an electrical finite element model. The great advantage of finite element methods (FEMs) over many other modelling techniques is that they are capable of modelling structures of high complexity, such as the cochlea. This ability has, for example, permitted accurate modelling of cochlear micro-mechanics ( $\mathrm{Ni}$ et al., 2016). Electrical FEMs have been used to study the behaviour of cochlear implants (Briaire and Frijns, 2000; Choi and Wang, 2014; Saba, 2012; Sue et al., 2013; Tran et al., 2011; Tran et al., 2013). Harland et al. (2015) present a very 


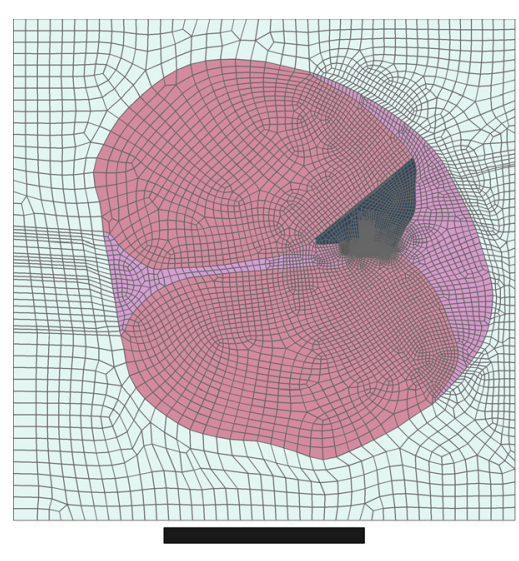

(a)

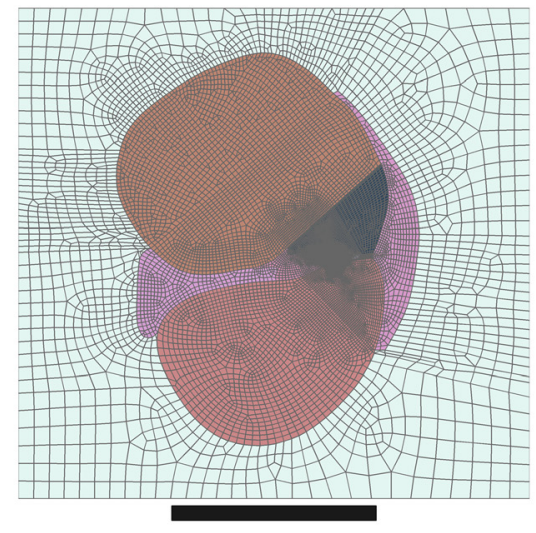

(b)

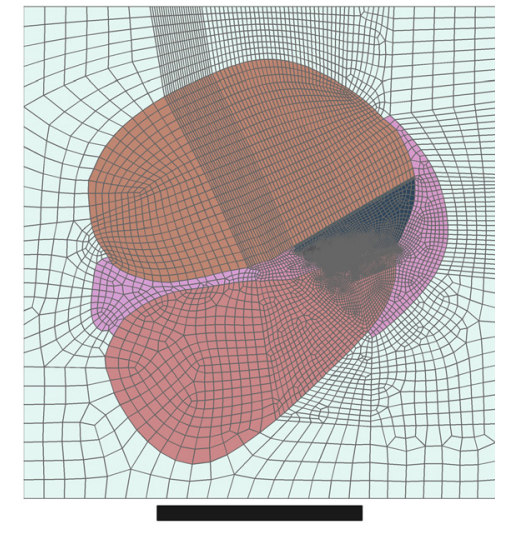

(c)

FIG. 1. (Color online) Meshed cochlea slices (a) $12 \mathrm{~mm}$ from the base, (b) $19 \mathrm{~mm}$ from the base, and (c) $24 \mathrm{~mm}$ from the base. The surrounding material is bone. The length bars indicate $1 \mathrm{~mm}$.

detailed model of OHC membranes. FEMs, however, do not appear to have been previously used to model the propagation of fields from hair cells.

\section{THE FINITE ELEMENT COCHLEA}

\section{A. Human cochlear geometry}

The cochlea is a multi-scale complex structure and its geometry is usually simplified for modelling purposes. We, however, have developed a set of detailed geometrical models of the human cochlea based on histological images obtained at different positions along the cochlea corresponding to different characteristic frequencies (Bellos et al., 2014), as shown in Fig. 1. In each model, the scala vestibuli and scala media are separated by the Reissner's membrane, and the organ of Corti, located between the scala media and scala tympani, contains receptor cells consisting of one row of inner hair cell and three rows of OHCs, as well as supporting cells including the pillar cells. The geometries were meshed into quadrilaterals as shown in Fig. 1, and a close-up view of the organ of Corti at $12 \mathrm{~mm}$ from the base is shown in Fig. 2.

The slices were then extruded so that each quadrilateral element becomes a hexahedron, as shown in Fig. 3. When the extrusion is performed, the hair cells were made to have approximately the same size in the radial and tangential dimensions. Three slices of hair cell elements are alternated with three slices of the supporting material elements. The extrusion was arranged to include the curvature of the cochlea, as shown in Fig. 3. The radius of curvature is approximately $3.8 \mathrm{~mm}$ at the base, decreasing to $1.2 \mathrm{~mm}$ at the apex (Yoo et al., 2000). This curvature was not found to cause any significant effect on the results. A limitation of the extrusion approach is that it cannot easily be used to analyse the effect of electrical propagation on the opposite side of the cochlea. The reason for this is because of the discontinuity that results at the centre of the rotation, analogous to a branch point encountered in complex analysis (Cohen, 2007). With sufficient elements, the extrusion approach could be used to model propagation between the turns on the same side of the cochlea. However (using the $12 \mathrm{~mm}$ slice as an example), the number of elements (8874) within each slice, and the number of nodes (8975) meant that computer memory was a limitation long before a complete turn of the cochlea could be modelled. The results presented are for extrusions of 57 elements along the tangential direction, leading a total of 505818 elements and 520550 nodes.

Convergence of the mesh is an important consideration. A full mesh-convergence analysis was not feasible with the computer memory available, but convergence was examined by varying the fineness of the mesh in the tangential direction. It was found that variation of the decay rate was not great after about three elements per row, which is the value used in the manuscript. The decay rate appeared to increase slightly with the finer mesh (the length constant decreased by at most $40 \%$ ), which is consistent with the results we discuss later.

Details of the electrostatic FEM technique are presented in Appendix A.

\section{B. Boundary conditions}

A critical factor in any physical modelling is the specification of boundary conditions. It is assumed that the

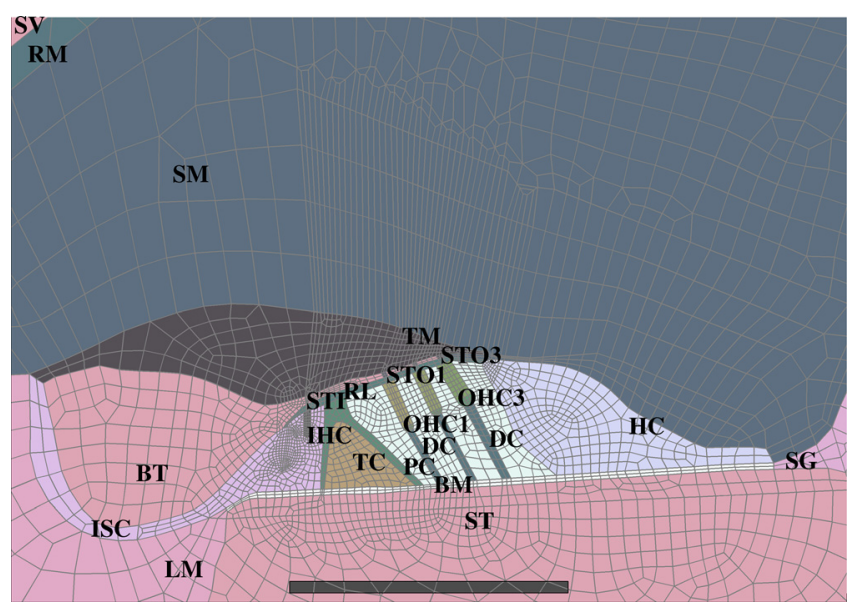

FIG. 2. (Color online) Close up the mesh cross section at $12 \mathrm{~mm}$ from the base. (SV) Scala vestibuli, (SM) scala media, (ST) scala tympani, (SG) spiral ligament, (PC) pillar cell, (IHC) inner hair cell, (ISC) inner sulcus cells, (TM) tectorial membrane, (RL) reticular lamina, (STOx) OHCs stereocilia, $(\mathrm{OHC} x$ ) OHCs, (HC) Hensen cells, (BM) basilar membrane, (STI) inner hair cell stereocilia, (LM) spiral limbus, (DC) Deiters cells, (RM) Reissner's membrane, (TC) tunnel of Corti, (BT) sub-tectorial space. The length bar indicates $100 \mu \mathrm{m}$. 


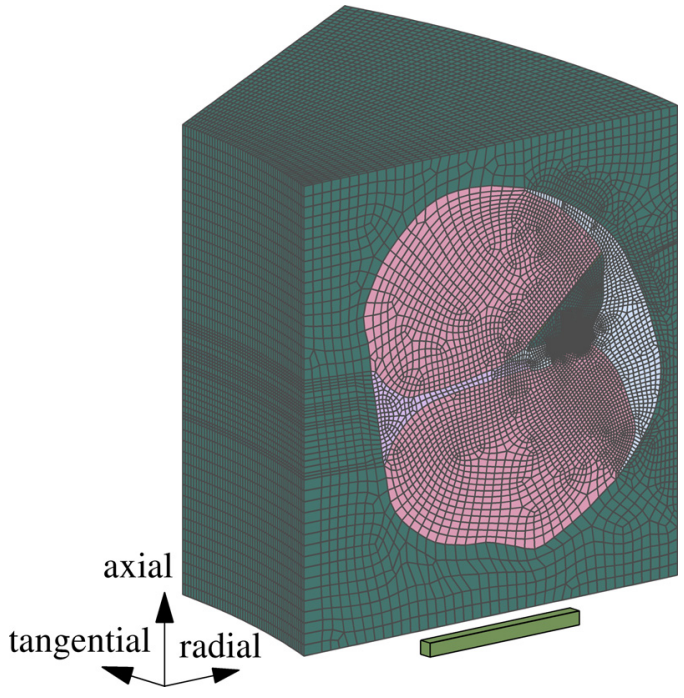

FIG. 3. (Color online) Extrusion of the cochlea slice to permit the modelling of longitudinal electrical coupling. Note that the tangential extent of the extrusion has been exaggerated in this illustration by a factor of approximately 20 to make the curvature more visible. The length bar indicates $1 \mathrm{~mm}$.

electrical potential at locations exterior to the cochlea is zero. Ideally this would be modelled by a boundary at a considerable distance, but due to the limitation of the available geometry and the number of elements, the zero voltage is located at the visible boundaries (i.e., the front, rear, upper and lower surfaces, and the two curved surfaces) seen in Fig. 3. For consideration of electrical interaction between hair cells that are near each other, this boundary condition was found to have negligible effect on the results. This was determined by locating an active row of hair cells at different positions within the simulated volume. The maximum deviation in the voltage decay rates estimated in this way was approximately $0.1 \%$. The effect is not negligible for the voltages in the scala vestibuli and scala tympani, but this does not alter the conclusions of the study.

The electrical excitation provided by the hair cells is assumed to take the form of a current. This was modelled by a time-varying dipole of charge, with the apex and base of the hair cell carrying equal and opposite charge. At the apex of the cell, the charged region was the elements representing the stereocilia. For the base, the charged region was all the hair cell elements below the reticular laminar. The charge density in each element was calculated by dividing the unit charge by the sum of the volumes of the elements corresponding to these two regions.

\section{Material properties}

The material properties used in the finite element model are listed in Table I. Some conductivities are from Briaire and Frijns (2000), who refer to Finley et al. (1990) and Suesserman (1992). Sue et al. (2013) cite Cantrell et al. (2008) as giving perilymph properties of conductance $\sigma=1420 \mathrm{mS} / \mathrm{m}$ and relative permittivity $\varepsilon_{\mathrm{r}}=78$. It has been assumed that inter-species variation in these properties is insignificant. Some helpful discussions of the variations between the various sources are presented in Saba (2012),
TABLE I. Electrical properties of cochlear material.

\begin{tabular}{lcccc}
\hline \hline Material & Conductivity (mS/m) & Permittivity (relative) \\
\hline Endolymph & 1680 & Strelioff (1973) & - & \\
Perilymph & 1420 & Strelioff (1973) & 78 & Cantrell et al. (2008) \\
Bone & 10 & Tran et al. (2013) & 800 & Kosterich et al. (1983) \\
Reissner's membrane & 2 & Saba (2012) & - & \\
Basilar membrane & 27 & Saba (2012) & - \\
Stria vascularis & 5.3 & Briaire and & - \\
& \multicolumn{5}{c}{ Frijns (2000) } \\
Organ of Corti & 12 & Briaire and & - \\
& \multicolumn{5}{c}{ Frijns (2000) } \\
\hline \hline
\end{tabular}

who refers to Finley et al. (1990), Briaire and Frijns (2000), and Rattay et al. (2001). Some additional material properties are provided by Dallos (1984), Tran et al. (2013), and Jia et al. (2007).

The material properties not included in the table are not known. These materials that were separately segmented, but for which information on electrical properties was unavailable were: the tectorial membrane, Deiters's cells, Hensen cells, pillar cells, inner sulcus cells, and the hair cells themselves (both inner and outer). These are components of the organ of Corti, and were thus assumed to have a conductivity of about $14 \mathrm{mS} / \mathrm{m}$ and a relative permittivity of about 78 . The spiral limbus and spiral lamina were given properties close to those of bone.

There were no two materials that were assigned exactly the same value, because some slight variation in properties would normally be expected to be present. Some experimentation was conducted in which the material properties were changed, some of them fairly significantly (variation of $50 \%$ from the initially specified values). In particular, the conductivity of the reticular lamina (one of the unknown properties) was subject to scrutiny because this is very near the hair cells. Although detectable, these variations did not alter any of the conclusions of this paper.

\section{Post-processing}

Modelling the current passing through an $\mathrm{OHC}$ as a time varying charge dipole results in the matrix equation

$$
F v_{\text {FEM }}=q,
$$

where $v_{\text {FEM }}$ is a vector of unknown node voltages and vector $q$ represents the element charges. We use a frequency domain implementation, and an assumed time dependence $e^{j \omega t}$ in the voltages and currents, where $t$ is time, $\omega$ is the angular frequency and $j=\sqrt{-1}$. The element charges are zero except for the elements of the active hair cells, for which $q=i_{\text {FEM }} /(j \omega)$ where $i_{\text {FEM }}$ is the current. The system matrix $F$ consists of real and imaginary parts,

$$
F=F_{\epsilon}-\frac{j}{\omega} F_{\sigma}
$$

where $F_{\epsilon}$ contains information about the permittivity of the materials, and $F_{\sigma}$ contains information about the conductivity of the materials. 
Thus Eq. (1) can be written as

$$
\left(F_{\epsilon}-\frac{j}{\omega} F_{\sigma}\right) v_{\mathrm{FEM}}=\frac{i}{j \omega},
$$

and multiplying by $j \omega$ gives

$$
\left(F_{\sigma}+j \omega F_{\epsilon}\right) v_{\mathrm{FEM}}=i_{\mathrm{FEM}} .
$$

It is shown in Appendix A that the corresponding elements of $\omega F_{\epsilon}$ are considerably smaller than those of $F_{\sigma}$, because they depend, respectively, on the real and imaginary parts of Eq. (A2). This means that the voltages $v_{\text {FEM }}$ can be obtained using a Taylor expansion of the matrix inverse:

$$
\begin{aligned}
v_{\mathrm{FEM}} & =\left(F_{\sigma}+j \omega F_{\epsilon}\right)^{-1} i_{\mathrm{FEM}} \\
& \approx\left(F_{\sigma}^{-1}-j \omega F_{\sigma}^{-1} F_{\epsilon} F_{\sigma}^{-1}\right) i_{\mathrm{FEM}} .
\end{aligned}
$$

The approximate frequency independence of $\Re\left(F^{-1}\right)$ and of $\Im\left(F^{-1} / \omega\right)$ has been empirically confirmed (the norm of the variations encountered when varying the frequency over the range $1 \mathrm{~Hz}$ to $20 \mathrm{kHz}$, when divided by the norm of the actual voltages, is less than $8 \times 10^{-4}$ ). This result means that the equation need not be solved separately for every frequency. In many cases, the first approximation $v_{\text {FEM }}=F_{\sigma}^{-1} i_{\text {FEM }}$ may be sufficiently accurate. Indeed, most studies of electrical effects in the scalae of the cochlea have assumed this result: that resistive effects dominate, and capacitive effects represented by $F_{\epsilon}$ can be ignored. But if more accuracy is desired, a second approximation based on Eq. (5) does not require much more computation. One way to perform this computation is to solve the matrix problem using a matrix $F$ based on a small value of frequency $\omega$ and scale the imaginary part of $v_{\text {FEM }}$ to obtain the result for other frequencies.

It may be desirable to compare the results of the finite element model with that of a lumped parameter model of electrical conduction along the scalae; we make this comparison in Sec. IV. For this we define a vector of currents through OHCs $i_{\mathrm{x}}$ and voltages across OHCs $v_{\mathrm{x}}$. The number of elements in these vectors will depend on the number of sections of the lumped parameter model used to represent the cochlea but is typically less than 1000 .

The vector $v_{\mathrm{x}}$ is derived from vector $v_{\mathrm{FEM}}$ by taking the difference between the average of the voltages of the nodes of the hair cell somata and the average of the nodes of the stereocilia. This operation is represented by matrix $C_{\mathrm{v}}$. The vector $i_{\text {FEM }}$ is calculated from $i_{\mathrm{x}}$ by distributing this current as time varying charge among the elements of the hair cells. This operation is represented by the matrix $C_{\mathrm{i}}$. Combining $v_{\mathrm{x}}=C_{\mathrm{v}} v_{\mathrm{FEM}}$ and $i_{\mathrm{FEM}}=C_{\mathrm{i}} i_{\mathrm{x}}$, we have

$$
v_{\mathrm{x}}=C_{\mathrm{v}} F^{-1} C_{\mathrm{i}} i_{\mathrm{x}}
$$

or equivalently

$$
i_{\mathrm{x}}=\left(C_{\mathrm{v}}\left(F_{\sigma}+j \omega F_{\epsilon}\right)^{-1} C_{\mathrm{i}}\right)^{-1} v_{\mathrm{x}},
$$

which, because of the small values in $F_{\epsilon}$, can be approximated as

$$
\begin{aligned}
i_{\mathrm{x}} \approx & \left(\left(C_{\mathrm{v}} F_{\sigma}^{-1} C_{\mathrm{i}}\right)^{-1}+j \omega\left(C_{\mathrm{v}} F_{\sigma}^{-1} C_{\mathrm{i}}\right)^{-1} C_{\mathrm{v}} F_{\sigma}^{-1} F_{\epsilon}\right. \\
& \left.\times F_{\sigma}^{-1} C_{\mathrm{i}}\left(C_{\mathrm{v}} F_{\sigma}^{-1} C_{\mathrm{i}}\right)^{-1}\right) v_{\mathrm{x}} \\
\approx & \left(C_{\mathrm{v}} F_{\sigma}^{-1} C_{\mathrm{i}}\right)^{-1} v_{\mathrm{x}} .
\end{aligned}
$$

The approximate frequency independence of $\Re\left(\left(C_{\mathrm{v}} F^{-1} C_{\mathrm{i}}\right)^{-1}\right)$ and of $\Im\left(\left(C_{\mathrm{v}} F^{-1} C_{\mathrm{i}}\right)^{-1} / \omega\right)$ has also been empirically confirmed to produce less error than $0.1 \%$ over the frequency range $1 \mathrm{~Hz}$ to $20 \mathrm{kHz}$.

\section{RESULTS}

\section{A. Finite element model}

The real part of the voltages resulting from unit current flowing through a single row of three hair cells is shown in Fig. 4 for the $12 \mathrm{~mm}$ slice. Contributions from the inner hair cell are ignored, and it is assumed that the three hair cells in the row operate in phase with each other.

Inner hair cell electrical activity could be modelled separately, and combined with the OHC activity, although the frequency and phase relationship between the two is strongly frequency dependent, and we have not done so here.

The OHCs can only be considered to be approximately in phase for both mechanical stimulation (Fridberger et al., 2002) and electrical stimulation (Nowotny and Gummer, 2011; Nuttall et al., 1999). The first and second rows of OHCs move in-phase with both acoustical and electrical excitations, as shown in Figs. 4 and 8 of Ni et al. (2016) and also observed by Karavitaki and Mountain (2007) in the excised cochlea with electrical stimuli. The third row moves not strictly in-phase with the other two rows when excited electrically, due to greater influence from the Hensen cells, as shown in Fig. 4 of $\mathrm{Ni}$ et al. (2016) and Fig. 12 of Karavitaki and Mountain (2007). Figure 4 of Ni et al. (2016) suggests that the phase variation is for combined mechanical and electrical stimulation is almost undetectable for frequencies below $1 \mathrm{kHz}$, but the variation between $\mathrm{OHC}$ row 1 and row 3 rises to about $15^{\circ}$ at $5 \mathrm{kHz}$.

The results for the 19 and $24 \mathrm{~mm}$ slices are qualitatively similar to those for the $12 \mathrm{~mm}$ slice. A feature of these results that is immediately apparent is that the voltage decays rapidly with distance, and so this feature of the results was studied in more detail. The effect induced by an electrical potential on a hair cell is assumed to be measurable as the potential difference between the voltage in the hair cell, and the voltage at the stereocilia. The voltages at the nodes representing each of these were averaged across the three hair cells in each row, and then the difference between the resulting two quantities obtained. This is presented versus the distance from the active row in Fig. 5(a). For separation between the hair cell rows of approximately $10 \mu \mathrm{m}$, the space constant appears to be approximately $11 \mu \mathrm{m}$ for the slice $12 \mathrm{~mm}$ from the base, and close to $20 \mu \mathrm{m}$ for the $19 \mathrm{~mm}$ and $24 \mathrm{~mm}$ slices. This is very different from the values in the range $0.5-4 \mathrm{~mm}$ assumed by Strelioff (1973) and the other authors listed in Sec. I, and measured by Johnston et al. (1966). The result is however much closer to the $42 \mu \mathrm{m}$ space constant reported by Fridberger et al. (2004), and the $80 \mu \mathrm{m}$ space constant reported by Dong and Olson (2013). 


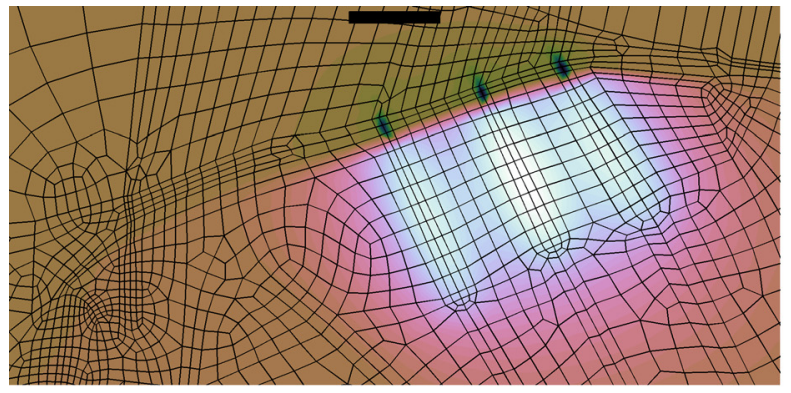

(a)

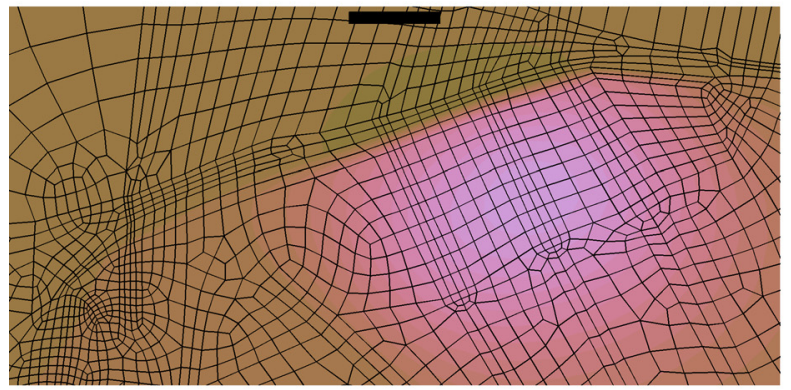

(c)

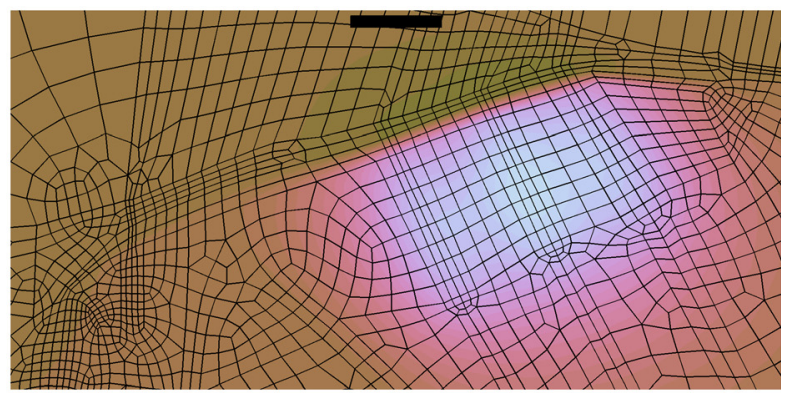

(b)

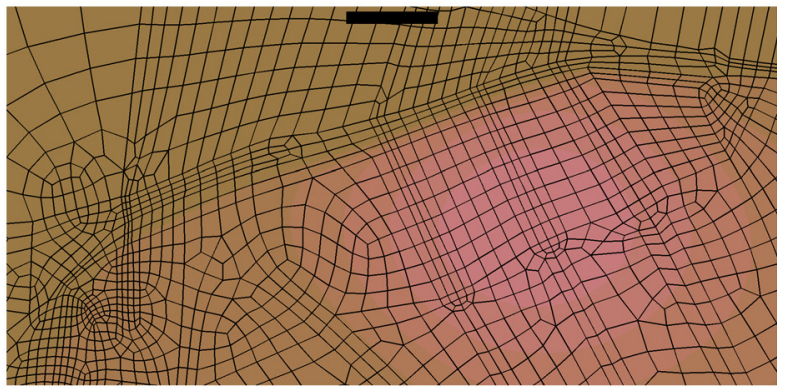

(d)

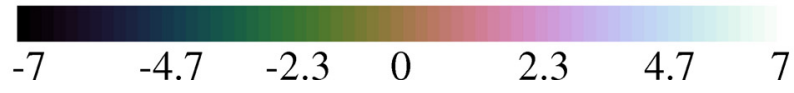

FIG. 4. (Color online) Voltages (a) in the plane of the active hair cell, (b) between the active and the adjacent hair cell row, (c) at one row distance and (d) at two rows distance. The colour maps are generated using the method of Green (2011) for viewing in colour or greyscale. The length bars indicate $10 \mu \mathrm{m}$. The scale is in kilovolts per ampere of hair cell current.

There is such a discrepancy between these results that some explanation is necessary, and the FEM can assist in providing this explanation.

The most immediate suggestion is that the proximity of the boundaries of the finite element model at $0 \mathrm{~V}$ are imposing an unrealistically high rate of decay. It is true that the effect of the boundary is not negligible and is apparent in Fig. 5, causing the last value to deviate from the decay rate seen closer to the active hair cells. The extent of this effect was investigated by making the active row asymmetrically located: the simulated volume includes nine rows of hair cells, and so if rows other than number five are active, the decay is somewhat asymmetrical. It was found that the change in decay is only significant in the row closest to the boundary. The space constant was not significantly influenced (variation of about $0.1 \%$ ) by the location of the boundary.

Another possibility is that the reticular laminar provides a significant insulating effect between the endolymph, in which the stereocilia are located, and the organ of Corti, in which the somata of the OHCs are located. By preventing electrical propagation between these two regions, the reticular lamina could extend the range of electrical propagation. As mentioned in Sec. IIC this possibility was investigated by decreasing the conductivity of the reticular laminar (from the default value of $12 \mathrm{mS} / \mathrm{m}$ ) to very low values. The effects were visible but did not appreciably change the space constant.

The real difference between the finite element model, and previous work, is that earlier models have assumed that currents that flow through the hair cells cause voltage changes across the entire cross section of the scalae, in order for the resistance values based on the scalae cross-sectional areas and conductivities to be relevant. Modelling the hair cells as current dipoles raises the possibility that this coupling into the scalae does not occur; the geometry of hair cell connection to the scalae becomes an important consideration.

\section{B. Simple dipole model}

In this section, we present results of a small investigation into the behaviour of a current dipole. This abstracts the situation somewhat from a realistic geometry but allows the essence of our understanding of the situation to be more easily understood.

A unit length dipole (representing the active hair cell) having unit (positive and negative) charges is placed at the origin. Another dipole (representing another hair cell) is placed at an arbitrary position near the first dipole, and aligned parallel to it. The voltage induced by the first dipole in the second was investigated by simply applying Coulomb's law. The effect of a grounded boundary sphere of radius five dipole lengths was also investigated using the method of image charges (Tikhonov and Samarskii, 1963). The grounded boundary condition of the finite element model is considerably further from the hair cells than this. The results are shown in Fig. 6 and it is apparent that the proximity of a grounded boundary condition has perceptible but insignificant effect on the voltage. It is also apparent that the decay is very rapid. The decay rate is shown in Fig. 7. 

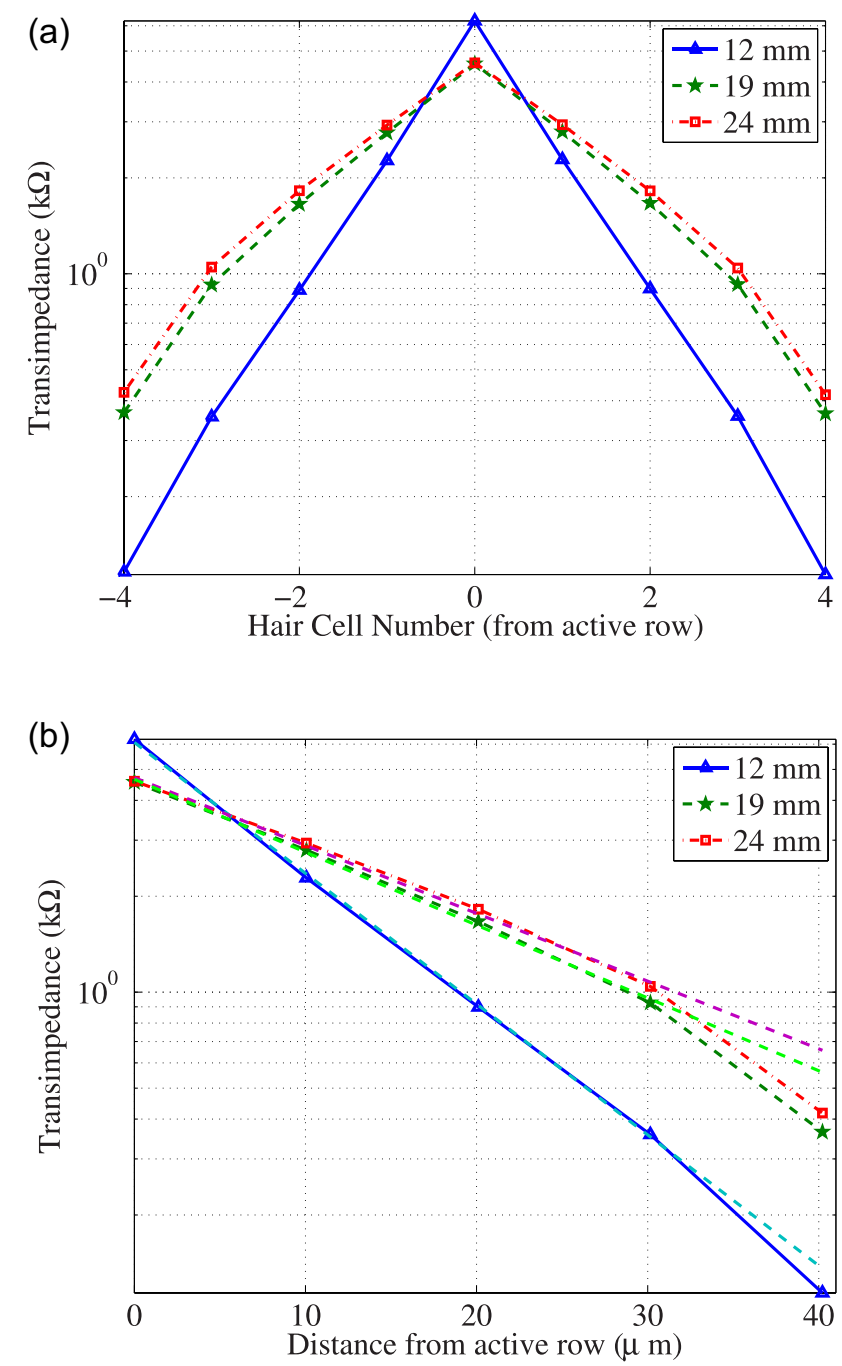

FIG. 5. (Color online) Voltage decay with distance from the active hair cell row. The dashed lines added in (b) are lines minimising the mean squared error for exponential decay. Since the response is the voltage per unit current, it has the dimensions of impedance.

The curves are not a good fit to an exponential decay; rather, the voltages decays inversely with distance as one would expect from a monopole charge (for the finer tangential mesh mentioned earlier in connection with convergence, the behaviour variation also showed this tendency). However, near the dipole, the space constant can be calculated as being about $0.1-0.4$ dipole lengths, depending on the relative positions of the two dipoles.

Although this simple study does not bear great resemblance to conditions inside the cochlea, it does show clearly that the strongest single factor influencing the range of influence of a hair cell modelled as a current dipole will be the length of the hair cell, and the space constant will be on the order of this length. OHCs increase in length towards the apex, but are typically in the range $25-45 \mu \mathrm{m}$. The implication of this is that the extent of electrical coupling within the cochlea may be much weaker than has been supposed in previous studies.

The voltage decay rate measured using electrodes inserted into the scalae as per Johnston et al. (1966) do not accurately reflect the range of influence of dipoles the size of
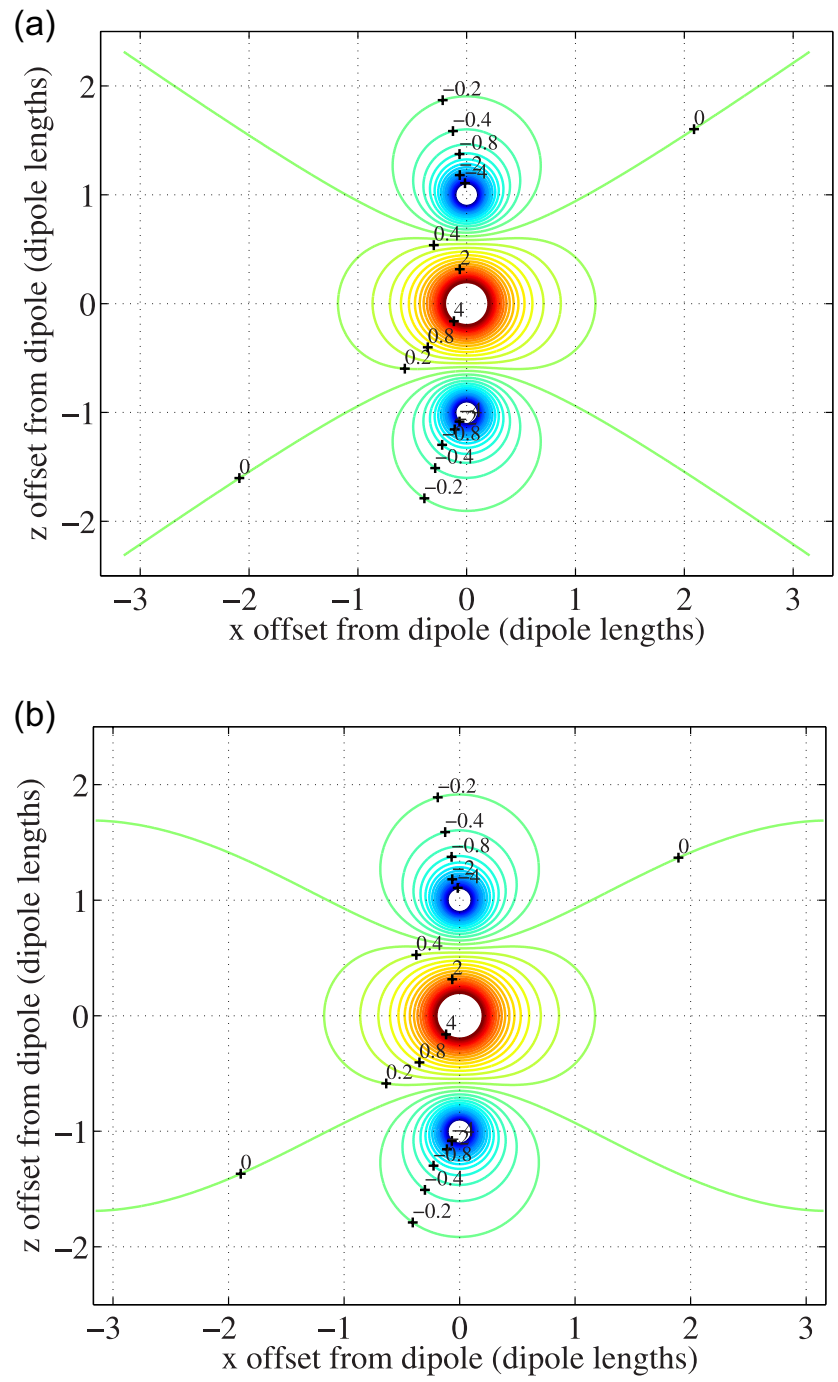

FIG. 6. (Color online) Voltage induced by a unit charge dipole at other dipole positions oriented parallel to the source dipole. The units are in dipole lengths. In (a) the boundary is at infinite distance, while in (b) a grounded sphere of radius five dipole lengths surrounds the dipole. It can be seen that the voltage decays rapidly with distance from the charged dipole, and that this conclusion is not altered by proximity of a boundary.

hair cells. Such electrodes will measure the resistance through a large cross section of perilymph or endolymph. Hair cells are prevented from coupling into the scalae in this way because they are essentially small current dipoles. The more recent measurements of Dong and Olson (2013) involve measurements closer to the actual location of hair cells, and more closely reflect the action of current dipoles.

\section{EQUIVALENT LUMPED PARAMETER MODEL}

The models that have been used to predict a greater range of influence (up to $4 \mathrm{~mm}$ ) of hair cell electrical activity are similar to that of Strelioff (1973). An interesting question is whether the parameters of such a model can be modified so as to produce results similar to the finite element model presented here. We consider here the model shown in Fig. 8. This model is very similar to that of Ramamoorthy et al. (2007), although the nomenclature of the resistance components is chosen to be similar to that of Strelioff (1973). An 


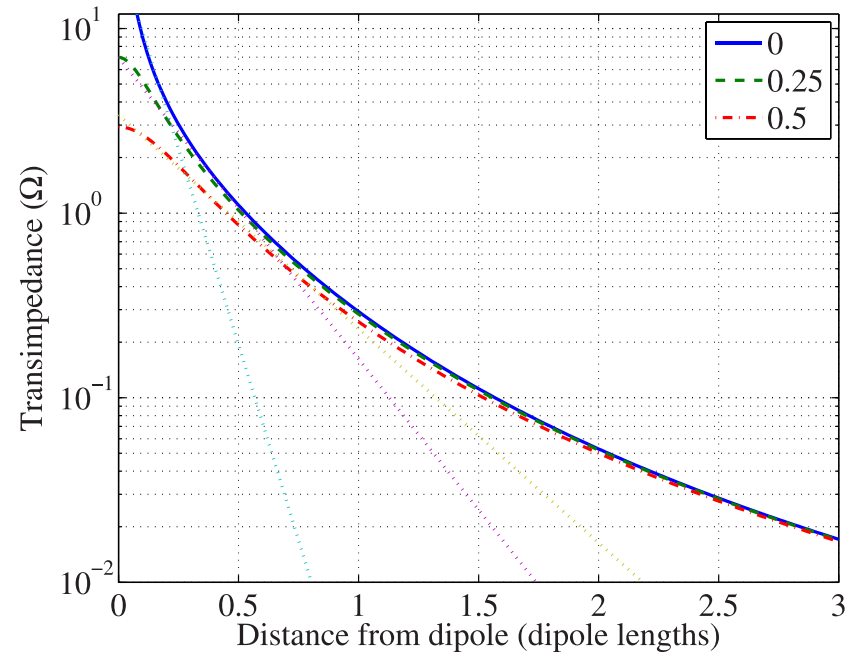

FIG. 7. (Color online) Decay rate for the voltage induced by a unit charge dipole at another dipole of the same length. The three curves are for different distances (measured in dipole lengths) from the perpendicular bisector of the source dipole. The dotted lines indicate exponential decays based on minimising the squared error of the first 1.5 wavelengths.

original feature of the model of Fig. 8 is that the electrical properties of the scalae have been separated from those of the hair cells. By analogy with models of cochlear mechanical behaviour, we call these two properties the macroelectrics and the micro-electrics, respectively. We do not consider the micro-electrics further here, but instead focus on the macro-electrics, shown on the left side of Fig. 8, as this is the part of the model that is functionally equivalent to the finite element model.

The circuit analysis of Fig. 8 is contained in Appendix $\mathrm{B}$, where it is shown that the voltages across the hair cells and current through the hair cells can be represented as

$$
Y v_{\mathrm{x}}=i_{\mathrm{x}}
$$
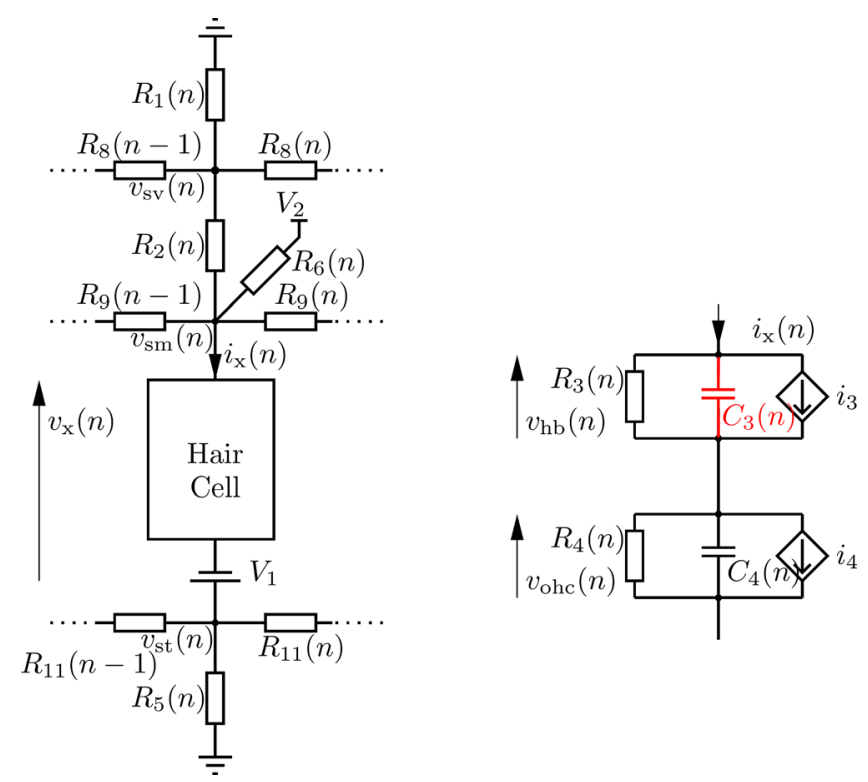

FIG. 8. (Color online) Separation of the model into macro-electrics (on the left) and micro-electrics (on the right). The macro-electrics can be arranged to provide an approximation to the functionality of the finite element model.

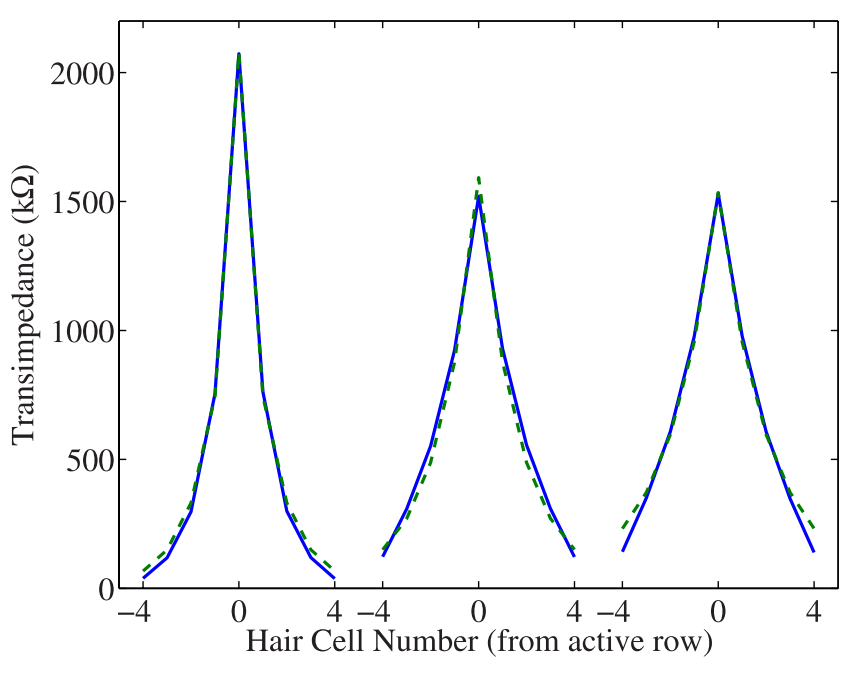

FIG. 9. (Color online) Comparison between the voltage resulting from unit hair-cell current in the finite element model (solid) and the lumped parameter model (dashed) at the three locations 12, 19, and $24 \mathrm{~mm}$ from the base. These results differ from those of Fig. 5 by a factor of three because there, the excitation consisted of three unit current dipoles, whereas here, the total current across the three hair cells is a single unit.

It is clear that Eq. (9) is essentially equivalent to Eq. (6), although the lumped parameter model only includes resistors at this stage. The slices used in the finite element model at 12, 19, and $24 \mathrm{~mm}$ from the base yield the values near the diagonal of three columns of the diagonally dominant matrix $Y$. The resistances $R_{1}(n), \ldots, R_{11}(n)$ were allowed to vary logarithmically along the length of the cochlea, parameterised by the values at $n=1$ (at the cochlea base) and $n=3500$ (at the cochlea apex). The scala tympani and scala vestibuli are effectively shorted together at the apex, while the scala media is an open circuit at the apex.

A simple search using the method of Nelder and Mead (1965) was used to find values of these resistances that minimised the sum of squared errors between results of the finite element and lumped models. The resulting similarity between the models is shown in Fig. 9. The result was found to be relatively insensitive to the values of $R_{1}, R_{2}$, and $R_{8}$ so these were left similar to the values of Strelioff (1973). The resulting parameters are listed in Table II. It can be seen that some of these values are far from those calculated on the basis of the conductivity of the lymph cross-sectional areas of the scalae; the least physiologically realistic value is the value of the scala media to ground resistance, that nearly vanishes at the apex. However, these model parameters yield electrical coupling very close to that of the finite element model. This is only one parameter set, and as the process of matching between the models is rather under-determined, it is possible that there are other parameter sets corresponding to local minima of the error function that could perform equally well in reconciling the two models. If more than three cochlear crosssections were available for the finite element model, the model-matching problem would be less under-determined, and the simple logarithmic variation used here could be replaced by a more flexible parameter variation.

It should be noted that in Dimitriadas and Chadwick (1999) the resistance that is here labelled $R_{11}$ does not 
TABLE II. Parameter set for the lumped parameter macro-electrics cochlea model. The values vary logarithmically between the two values given for the base and apex. To obtain the values for a lumped model the "lateral" resistances $R_{1}, \ldots, R_{6}$ should be divided by the length of one section, whereas the "longitudinal" resistances $R_{8}, \ldots, R_{11}$ should be multiplied by the length of one section. The "classical" values are from Ramamoorthy et al. (2007), based on Strelioff (1973), although the value of $R_{6}$ is directly from Strelioff (1973); $R_{6}$ is effectively infinite in Ramamoorthy et al. (2007).

\begin{tabular}{|c|c|c|c|c|c|}
\hline & Base & Apex & Units & Description & "Classical" \\
\hline$R_{1}$ & 5 & 15 & $\Omega \mathrm{m}$ & Scala vestibuli to ground & 10 \\
\hline$R_{2}$ & 30 & 25 & $\Omega \mathrm{m}$ & Scala vestibuli to scala media & 25 \\
\hline$R_{5}$ & 0.028 & 0.098 & $\Omega \mathrm{m}$ & Scala tympani to ground & 4 \\
\hline$R_{6}$ & 3 & $2 \times 10^{-8}$ & $\Omega \mathrm{m}$ & Scala media to ground & 27 \\
\hline$R_{8}$ & 1 & 6 & $\mathrm{M} \Omega / \mathrm{m}$ & $\begin{array}{c}\text { Scala vestibuli longitudinal } \\
\text { resistance }\end{array}$ & 3 \\
\hline$R_{9}$ & 2000 & 2000 & $\mathrm{M} \Omega / \mathrm{m}$ & $\begin{array}{l}\text { Scala media longitudinal } \\
\text { resistance }\end{array}$ & 5 \\
\hline$R_{11}$ & 550 & 83 & $\mathrm{M} \Omega / \mathrm{m}$ & $\begin{array}{l}\text { Scala tympani longitudinal } \\
\text { resistance }\end{array}$ & 0.15 \\
\hline
\end{tabular}

represent the scala tympani, but a conductive pathway between OHCs and Dieter's cells (Zwislocki et al., 1992).

\section{CONCLUSION}

The finite element model presented has several deficiencies. On the one hand, it is not large enough to model the entire cochlea, and hence cannot model the possibility of coupling between different turns of the scalae, nor can it model coupling to an externally located electrode. On the other hand, it is not detailed enough to accurately model the detail of the hair cells and hair cell membranes. However, for the purpose of modelling the range of electrical influence of hair cells, the model is adequate. The model suggests that the decay of electrical potential with distance from a hair cell is much more rapid than has been considered the case in several previous studies based on measurements or on modelling the resistance of the scalae. At first, this result may be surprising, but on closer consideration can be seen to be the natural result of the small size of the hair cells, which neither the earlier models nor the measurements using electrodes in the scalae consider. The new results are more consistent with the more recent measurements made closer to the hair cells of Fridberger et al. (2004) and Dong and Olson (2013).

It is possible that ion diffusion, such as investigated in Zwislocki et al. (1992) have a longer range effect than electric field propagation. This could account for some of the discrepancy between experiments that have measured the length constant [the measurements of Johnston et al. (1966) used current pulse of several seconds duration]. However, such ion diffusion would necessarily operate at a much longer time scale than the (virtually instantaneous) electric field, and so its effect at high frequencies is likely to be small.

Much longer ranges of hair cell electrical influence have been considered to be the cause of the broadness of tuning of the cochlear microphonic (Patuzzi, 1987). However, it was shown in Ayat et al. (2015) that longitudinal coupling is not required for the cochlear microphonic to have broad tuning curves; rather the broadness is due to phase cancellation between the hair cell and hair bundle voltages. The shorter range of hair cell electrical influence proposed here is consistent with these earlier observations.

Although it has not been shown here, the finite element model can be readily extended to include the effects of inner hair cell activity. The relative phase and amplitude of the $\mathrm{OHC}$ and inner hair cell contributions are frequency dependent, and this would need to be taken into account if the two contributions were to be considered jointly.

It is important to note that the electrical coupling effects modelled in this paper are entirely independent of cochlear mechanics. However, the existence of bidirectional coupling between electrical and mechanical phenomena mean that the results of this investigation could have implications for cochlea physiology. The results of the finite element model have been summarised in a much more simple lumped parameter model, and this makes the results much more accessible for future studies of the effects of electrical coupling within the cochlea.

\section{ACKNOWLEDGMENT}

This research was conducted as part of the European FP7 SIFEM project, 600933. The authors would like to acknowledge the support of this project, and in particular, the slice geometries and the procedure for creating them that were developed as part of this project.

\section{APPENDIX A: ELECTROSTATIC FEM}

Many studies that apply FEM use commercial software. In the current study, however, the method was entirely implemented from first principles. The principles of acoustical FEMs will be familiar to many JASA readers, but the formulation for electrical studies may be less so, although the formulations for acoustical and electrical studies are very similar. This section presents a very brief overview of the approach used and its limitations. The primary sources used in the implementation were Zienkiewicz et al. (2013) and Humphries (2010).

A first step is the determination of whether radiation effects must be considered in the model. These can be ignored if the wavelength is very much greater than the system size, using what is called the low-frequency approximation. Using $L$ for the largest linear dimension of the system, $\omega$ for the angular frequency, $\epsilon$ for material permittivity, and $\mu$ for material permeability, this requirement can be expressed as

$$
\frac{L \omega}{2 \pi} \sqrt{\epsilon \mu} \ll 1 .
$$

Recall that the goal of this investigation is to study the effects of the electrical potentials at audio frequencies. At these frequencies, and for the relevant materials (the actual material properties are presented in Sec. IIC) the largest value obtained for the left of Eq. (A1) is approximately $2 \times 10^{-5}$, and therefore this low-frequency approximation is indeed valid. 
There are no known nonlinear effects associated with electrical field propagation in the cochlea.

We use a frequency domain implementation, where the voltages and currents have an assumed time dependence $e^{j \omega t}$ where $t$ is time, $\omega$ is the angular frequency and $j=\sqrt{-1}$. The model resembles an electrostatic one, with conductivity $\sigma$ modelled as the imaginary part of a complex permittivity

$$
\epsilon=\epsilon_{\mathrm{r}} \epsilon_{0}-j \frac{\sigma}{\omega}
$$

where $\epsilon_{0}$ is the permittivity of free space and $\epsilon_{\mathrm{r}}$ is the material relative permittivity. At a frequency of $20 \mathrm{kHz}$, the ratio of the real part to the imaginary part of this permittivity for bone is 0.089 . For other materials and lower frequencies the ratio is smaller. This means that although capacitive effects are not negligible, they are small compared to the resistive effects. This leads to the simplifying approximations used in Sec. II D.

In electrostatic finite element modelling the key unknown is voltage. The Euler-Lagrange approach to formulating the finite element equations is to use hypothesised values of the voltages $\phi$ to infer the electric field $(\mathbf{E}=-\nabla \phi$ where $\nabla$ represents the gradient operator). From this the electrostatic energy density $u_{\mathrm{e}}=\epsilon E^{2} / 2$ can be calculated, where $E$ is the magnitude of vector $\mathbf{E}$. The Euler-Lagrange functional is the total energy, given by the volume integral of $u_{\mathrm{e}}$, thus given by

$$
L=\iiint\left(\frac{\epsilon}{2}\left(\left(\frac{\partial \phi}{\partial x}\right)^{2}+\left(\frac{\partial \phi}{\partial y}\right)^{2}+\left(\frac{\partial \phi}{\partial z}\right)^{2}\right)-\rho_{\text {fixed }} \phi\right) d V,
$$

where $\rho_{\text {fixed }}$ denotes prescribed space charge.

The principle of stationary action requires that the derivative of this functional with respect to voltage is 0 , and it is this condition that enables a system of linear equations to be formed, and the voltages calculated.

The FEM facilitates the equation formation for a complex system by approximating the energy density integral using shape functions defined on each element, and by evaluating the voltages only at a finite number of nodes, usually defined at vertices or edges of the elements (although there can also be nodes in the interior of elements if greater accuracy is required).

In the present study, basic eight-node hexahedral elements were used. The eight shape functions of the form $N_{i}$ $=\left(1 \pm \xi_{1}\right)\left(1 \pm \xi_{2}\right)\left(1 \pm \xi_{3}\right), i \in\{1, \ldots, 8\}$ are defined for cubes using local coordinates $(-1) \leq \xi_{1}, \xi_{2}, \xi_{3} \leq 1$. The required global derivatives (on the general hexahedra) using coordinates $x, y$, and $z$ are obtained from derivatives with respect to the local coordinates (on the cubes) using

$$
\nabla_{x} \phi=J^{-1} \nabla_{\xi} \phi
$$

where the $3 \times 3$ Jacobian matrix $J$ contains the derivatives with respect to $\xi_{1}, \xi_{2}$, and $\xi_{3}$ (the rows) of $x, y$, and $z$ (the columns). The integral for each element is approximated using Gaussian quadrature with weights $w_{\ell}, w_{m}$, and $w_{n}$ on the interval $[-1,1]$ and again transformed to global coordinates using the Jacobian:

$$
\begin{aligned}
& \iiint g(x, y, z) d x d y d z \\
& \quad=\int_{-1}^{1} \int_{-1}^{1} \int_{-1}^{1} \tilde{g}\left(\xi_{1}, \xi_{2}, \xi_{3}\right) J\left(\xi_{1}, \xi_{2}, \xi_{3}\right) d \xi_{1} d \xi_{2} d \xi_{3} \\
& \quad \approx \sum_{\ell} \sum_{m} \sum_{n} \tilde{g}\left(\xi_{1 \ell}, \xi_{2 m}, \xi_{3 n}\right) J\left(\xi_{1 \ell}, \xi_{2 m}, \xi_{3 n}\right) w_{\ell} w_{m} w_{n} .
\end{aligned}
$$

The resulting sparse system of equations is solved by first creating the incomplete LU factorisation of the FEM matrix to use as a preconditioner, and then using the generalised minimum residual iterative method (Saad, 2003).

The correctness of the implementation was validated for known geometries such as cylinders, for which analytic solutions are available.

\section{APPENDIX B: LUMPED MODEL DERIVATIONS}

The circuit of Fig. 8 can be analysed using the nodevoltage approach, yielding the following equations:

At $v_{\mathrm{sv}}$,

$$
\begin{aligned}
& -\frac{v_{\mathrm{sv}}(n-1)}{R_{8}(n-1)} \\
& \quad+v_{\mathrm{sv}}(n)\left(\frac{1}{R_{1}(n)}+\frac{1}{R_{2}(n)}+\frac{1}{R_{8}(n-1)}+\frac{1}{R_{8}(n)}\right) \\
& \quad-\frac{v_{\mathrm{sm}}(n)}{R_{2}(n)}-\frac{v_{\mathrm{sv}}(n+1)}{R_{8}(n)}=0 .
\end{aligned}
$$

At $v_{\mathrm{sm}}$,

$$
\begin{aligned}
& -\frac{v_{\mathrm{sm}}(n-1)}{R_{9}(n-1)}-\frac{v_{\mathrm{sv}}(n)}{R_{2}(n)}+v_{\mathrm{sm}}(n)\left(\frac{1}{R_{2}(n)}+\frac{1}{R_{6}(n)}\right. \\
& \left.+\frac{1}{R_{9}(n-1)}+\frac{1}{R_{9}(n)}\right)-\frac{v_{\mathrm{sm}}(n+1)}{R_{9}(n)}=\frac{V_{2}(n)}{R_{6}(n)}-i_{\mathrm{x}}(n) .
\end{aligned}
$$

At $v_{\text {st }}$

$$
\begin{aligned}
& -\frac{v_{\mathrm{st}}(n-1)}{R_{11}(n-1)}+v_{\mathrm{st}}(n)\left(\frac{1}{R_{11}(n-1)}+\frac{1}{R_{11}(n)}\right. \\
& \left.+\frac{1}{R_{5}(n)}\right)-\frac{v_{\mathrm{st}}(n+1)}{R_{11}(n)}=i_{\mathrm{x}}(n),
\end{aligned}
$$

and across the hair cell,

$$
v_{\mathrm{sm}}(n)=v_{\mathrm{st}}(n)+v_{\mathrm{x}}(n)+V_{1}(n) .
$$

These equations can then be represented using a matrix with diagonal blocks, 


$$
G_{\mathrm{MAC}} v_{\mathrm{MAC}}=i_{\mathrm{MAC}}
$$

Assuming we have only linear perturbations about the DC values, we can assume that $V_{1}(n)=V_{2}(n)=0$, and can further write that

$$
i_{\mathrm{MAC}}=D_{\mathrm{i}} i_{\mathrm{x}}
$$

where $i_{\mathrm{x}}=\left(i_{\mathrm{x}}(1), i_{\mathrm{x}}(2), \ldots, i_{\mathrm{x}}(N)\right)^{T}$ and

$$
D_{\mathrm{i}}=I_{N} \otimes(0, \quad-1, \quad 1)^{T}
$$

where $\otimes$ represents the Kronecker product.

We also have

$$
v_{\mathrm{x}}(n)=v_{\mathrm{sm}}(n)-v_{\mathrm{st}}(n),
$$

and so

$$
v_{\mathrm{x}}=D_{\mathrm{v}} v_{\mathrm{MAC}},
$$

where $v_{\mathrm{x}}=\left(v_{\mathrm{x}}(1), v_{\mathrm{x}}(2), \ldots, v_{\mathrm{x}}(N)\right)^{T}$ and

$$
D_{\mathrm{v}}=I_{N} \otimes\left(\begin{array}{lll}
0, & 1, & -1
\end{array}\right) .
$$

Combining these equations, we can write

$$
v_{\mathrm{x}}=D_{\mathrm{v}} G_{\mathrm{MAC}}^{-1} D_{\mathrm{i}} i_{\mathrm{x}},
$$

or equivalently

$$
Y v_{\mathrm{x}}=i_{\mathrm{x}} .
$$

Ayat, M., and Teal, P. (2013). "Modelling the generation of the cochlear microphonic," in IEEE International Conference on Medicine and Biology, Osaka, Japan, pp. 7168-7171.

Ayat, M., Teal, P. D., and McGuiness, M. (2014). "An integrated electromechanical model for the cochlear microphonic," Biocybernetics Biomed. Eng. 34(4), 206-209.

Ayat, M., Teal, P. D., Searchfield, G., and Razali, N. (2015). "Cochlear microphonic broad tuning curves," in Mechanics of Hearing: Protein to Perception, edited by K. Karavitaki and D. Corey (AIP, Melville, NY), pp. 1-6.

Bellos, C., Rigas, G., Spiridon, I., Bibas, A., Iliopoulou, D., Böhnke, F., Koutsouris, D., and Fotiadis, D. (2014). "Reconstruction of cochlea based on micro-CT and histological images of the human inner ear," BioMed Res. Int. 2014, 485783.

Briaire, J., and Frijns, J. (2000). "3D mesh generation to solve the electrical volume conduction problem in the implanted inner ear," Simulation Practice Theory 8, 57-73.

Cantrell, D., Inayat, S., Taflove, A., Ruoff, R., and Troy, J. (2008). "Incorporation of the electrode-electrolyte interface into finite-element models of metal microelectrodes," J. Neural Eng. 5(1), 54-67.

Chertoff, M., Earl, B., Diaz, F., and Sorensen, J. (2012). "Analysis of the cochlear microphonic to a low-frequency tone embedded in filtered noise," J. Acoust. Soc. Am. 132(5), 3351-3362.

Choi, C., and Wang, S. (2014). "Modeling ECAP in cochlear implants using the FEM and equivalent circuits," IEEE Trans. Magn. 50(2), 49-52.

Cohen, H. (2007). Complex Analysis with Applications in Science and Engineering (Springer, New York), pp. 165-174.

Dallos, P. (1984). "Some electrical circuit properties of the organ of Corti. ii. analysis including reactive elements," Hearing Res. 14, 281-291.

Dallos, P., and Evans, B. (1995). "High-frequency motility of outer hair cells and the cochlear amplifier," Science 267(5206), 2006-2009.

Dimitriadas, E., and Chadwick, R. (1999). "Solution of the inverse problem for a linear cochlear model: A tonotopic cochlear amplifier," J. Acoust. Soc. Am. 106(4), 1880-1892.
Dong, W., and Olson, E. (2013). "Detection of cohlear amplification and its activation," Biophys. J. 105(4), 1067-1078.

Finley, C., Wilson, B., and White, M. (1990). "Models of neural responsiveness to electrical stimulation," in Cochlear Implants: Models of the Electrically Stimulated Ear, edited by J. Miller and F. Spelman (Springer, New York), pp. 55-96.

Fridberger, A., de Monvel, J. B., and Ulfendahl, M. (2002). "Internal shearing within the hearing organ evoked by basilar membrane motion," J. Neurosci. 22, 9850-9857.

Fridberger, A., de Monvel, J., Zheng, J., Hu, N., Zou, Y., and Ren, T. (2004). "Organ of Corti potentials and the motion of the basilar membrane," J. Neurosci. 24, 10057-10063.

Geisler, C., Yates, G., Patuzzi, R., and Johnstone, B. (1990). "Saturation of outer hair cell receptor currents causes two-tone suppression," Hearing Res. 44(2-3), 241-256.

Green, D. (2011). "A colour scheme for the display of astronomical intensity images,” Bull. Astron. Soc. India 39, 289-295.

Harland, B., Lee, W., Brownell, W., Sun, S., and Spector, A. (2015). "The potential and electric field in the cochlear outer hair cell membrane," Med. Biol. Eng. Comput. 53, 405-413.

Honrubia, V., Strelioff, D., and Ward, P. (1973). "A quantitative study of cochlear potentials along the scala media of the guinea pig," J. Acoust. Soc. Am. 54(3), 600-609.

Humphries, S., Jr. (2010). Finite-Element Methods for Electromagnetics (Stanley Humphries, Albuquerque, NM), pp. 40-42.

Jia, S., Dallos, P., and He, D. (2007). "Mechanoelectric transduction of adult inner hair cells," J. Neurosci. 27(5), 1006-1014.

Johnston, B., Johnston, J., and Pugsley, I. (1966). "Membrane resistance in endolymphatic walls of the first turn of the guina-pig cochlea," J. Acoust. Soc. Am. 40(6), 1398-1404.

Karavitaki, K., and Mountain, D. (2007). "Imaging electrically evoked micromechanical motion within the organ of Corti of the excised gerbil," Biophys. J. 92(9), 3294-3316.

Kosterich, J., Foster, K., and Pollack, S. (1983). "Dielectric permittivity and electrical conductivity of fluid saturated bone," IEEE Trans. Biomed. Eng. 30(2), 81-86.

Mistrík, P., Mullaley, C., Mammano, F., and Ashmore, J. (2009). "Threedimensional current flow in a large-scale model of the cochlea and the mechanism of amplification of sound," J. R. Soc. Interface 6(32), 279-291.

Nelder, J., and Mead, R. (1965). "A simplex method for function minimization," Comput. J. 7, 308-313.

Ni, G., Elliott, S., and Baumgart, J. (2016). "Finite-element model of the active organ of Corti,” J. R. Soc. Interface 13, 20150913.

Nowotny, M., and Gummer, A. (2011). "Vibration responses of the organ of Corti and the tectorial membrane to electrical stimulation," J. Acoust. Soc. Am. 130(6), 3852-3872.

Nuttall, A., Guo, M., and Ren, T. (1999). "The radial pattern of basilar membrane motion evoked by electric stimulation of the cochlea," Hearing Res. 131, 39-46.

Patuzzi, R. (1987). "A model of the generation of the cochlear microphonic with nonlinear hair cell transduction and nonlinear basilar membrane mechanics," Hearing Res. 30, 73-82.

Ramamoorthy, S., Deo, N., and Grosh, K. (2007). "A mecho-electro-acoustical model for the cochlea: Response to acoustic stimuli," J. Acoust. Soc. Am. 121(5), 2758-2772.

Rattay, F., Leao, R., and Felix, H. (2001). "A model of the electrically excited human cochlear neuron. ii. influence of the three-dimensional cochlear structure on neural excitability," Hearing Res. 153, 64-79.

Saad, Y. (2003). Iterative Methods for Sparse Linear Systems (SIAM, Philadelphia, PA), pp. 171-194.

Saba, R. (2012). "Cochlear implant modelling: Stimulation and power consumption," Ph.D. thesis, University of Southampton, Southampton, UK, pp. 167-171.

Strelioff, D. (1973). "A computer simulation of the generation and distribution of cochlear potentials," J. Acoust. Soc. Am. 54(3), 620-629.

Sue, A., Tran, P., Wong, P., Li, Q., and Carter, P. (2013). "Time-domain finite element models of electrochemistry in intracochlear electrodes," in 35th Annual International Conference of the IEEE Engineering in Medicine and Biology Society, Osaka, Japan, pp. 1554-1557.

Suesserman, M. (1992). "Noninvasive microelectrode measurement technique for performing quantitative, in vivo measurements of the inner ear tissue impedances," Ph.D. thesis, University of Washington, Seattle, WA. 
Teal, P., Lineton, B., and Elliott, S. (2011). "An electromechanical model for the cochlear microphonic," in What Fire is in Mine Ears: Progress in Auditory Biomechanics, edited by C. Shera and E. Olson (AIP, Melville, NY), pp. 652-657.

Tikhonov, A., and Samarskii, A. (1963). Equations of Mathematical Physics (Pergamon Books, Oxford, UK), pp. 459-460.

Tran, P., Li, Q., and Carter, P. (2011). "Finite element modeling of current flow from cochlear implant stimulation," in Computational Intelligence and Bioinformatics: Modelling, Simulation, and Identification (International Association of Science and Technology for Development, Pittsburgh, PA), no. 755-053.

Tran, P., Wong, P., Sue, A., Li, Q., and Carter, P. (2013). "Influence of blood vessel conductivity in cochlear implant stimulation using a finite element head model," in 35th Annual International Conference of the IEEE Engineering in Medicine and Biology Society, Osaka, Japan, pp. 5291-5294.

Wever, E., and Bray, C. (1930). "Action currents in the auditory nerve in response to acoustic stimulation," Proc. Nat. Acad. Sci. U.S.A. 16, 344-350.

Yoo, S., Wang, G., Rubenstein, J., and Vannier, M. (2000). "Three-dimensional geometric modeling of the cochlea using helico-spiral approximation," IEEE Trans. Biomed. Eng. 47(10), 1392-1402.

Zienkiewicz, O., Taylor, R., and Zhu, J. (2013). The Finite Element Method: Its Basis and Fundamentals (Butterworth-Heinemann, Oxford, UK), pp.165-183.

Zwislocki, J., Slepecky, N., Cefaratti, L., and Smith, R. (1992). "Ionic coupling among cells in the organ of Corti," Hearing Res. 57(2), 175-194. 